\title{
Polymorphisms and haplotypes of IL2RA, IL-10, IFN- $\gamma$, IRF5, and CCR2 are associated with Epstein-Barr virus-associated hemophagocytic lymphohistiocytosis in children
}

\author{
Nannan Tang ${ }^{1}$, Junbin Huang ${ }^{1}$, Chun $\mathrm{Chen}^{1}$, Xiaojun $\mathrm{Wu}^{2}$, Hong $\mathrm{Xu}^{3}$, Guohua Chen ${ }^{4}$, and \\ Hongman Xue ${ }^{1}$ \\ ${ }^{1}$ The Seventh Affiliated Hospital Sun Yat-sen University \\ ${ }^{2}$ Sun Yat-sen Memorial Hospital, Sun Yat-sen University, Department of Pediatrics \\ ${ }^{3}$ The 2 nd affiliated hospital of Sun Yat-Sen university \\ ${ }^{4}$ Huizhou First Hospital
}

September 28, 2020

\begin{abstract}
Objective: Cytokine storms are central to the development of Epstein-Barr virus-associated hemophagocytic lymphohistiocytosis (EBV-HLH). Previous studies showed that single nucleotide polymorphisms (SNPs) of cytokine genes may be associated with the development of EBV-HLH in children. We investigated the associations between SNPs and haplotypes of interleukin-2 receptor subunit alpha (IL2RA), interleukin-10 (IL-10), interferon gamma (IFN- $\gamma$ ), IFN regulatory factor 5 (IRF5), and C$\mathrm{C}$ chemokine receptor 2 (CCR2) and susceptibility to EBV-HLH in children. Methods: 66 children with EBV-HLH and 58 healthy EBV-seropositive controls were enrolled in the study. SNPs of IL2RA rs2104286, rs12722489, and rs11594656, IL-10 rs1800896, rs1800871, and rs1800872, IFN- $\gamma$ rs2430561, IRF5 rs2004640, and CCR2 rs1799864 were assayed and genotyped using the SNaPshot technique. Results: The frequencies of the AA genotype and A allele of IL2RA rs2104286 and IL-10 rs1800896, and the CC genotype and C allele of IL-10 rs1800872 were significantly higher in the EBV-HLH group compared with those in the control group, respectively. The frequencies of genotypes and alleles of IL2RA rs2104286, IL-10 rs1800871, IFN- $\gamma$ rs2430561, IRF5 rs2004640, and CCR2 rs1799864 were similar in both groups. In addition, the IL2RA AGT (rs2104286rs12722489-rs11594656) and IL-10 ACC (rs1800896-rs1800871-rs1800872) haplotypes were also significantly more frequent in the EBV-HLH group. Conclusions: The SNPs of IL2RA rs2104286, IL-10 rs1800896 and rs1800872 and the haplotypes of IL2RA AGT and IL-10 ACC are highly associated with susceptibility to EBV-HLH in children.
\end{abstract}

\section{Materials and methods}

\section{Clinical data}

Sixty-six children with EBV-HLH diagnosed and treated at the Seventh Affiliated Hospital of Sun Yat-Sen University, Sun Yat-Sen Memorial Hospital of Sun Yat-Sen University, and Huizhou First Hospital from 2008 to 2019 were included in the study (EBV-HLH group; 28 males and 38 females, age 1.5-17 years, median age 5 years). Fifty-eight healthy children diagnosed as EBV-seropositive during the same period were included as the control group (32 males and 26 females, age 1-16.7 years, median age 4.3 years).

The inclusion criteria for EBV-HLH subjects were: (i) a diagnosis of HLH and exclusion of congenital $\mathrm{HLH}^{[6]}$, and (ii) diagnosis of EBV infection (positive for EBV antibodies or significantly increased EBVDNA in tissues or peripheral blood).

\section{Selection of SNPs}


SNPs retrieved from the HapMap database (http://www.hapmap.org/) were imported into Haploview 4.2 software (Broad Institute, Cambridge, MA, USA) and SNPs with a minor allele frequency (MAF) [?]0.01 were selected. IL2RA rs2104286, rs12722489, and rs11594656,IL-10 rs1800896, rs1800871, and rs1800872, $I \Phi N-\gamma \mathrm{rs} 2430561, I R F 5$ rs2004640, and CCR2 rs1799864 were finally identified for further study.

\section{Genotyping}

DNA was extracted from 1-2 ml peripheral whole blood using a blood DNA extraction kit (Tiangen, China) according to the manufacturer's directions. Gene polymorphism typing was performed using a SNaPshot Multiplex Kit (Thermo Fisher Scientific, Applied Biosystems, Foster City, CA ) according to the manufacturer's directions.

All the primers were designed using Primer Premier 6.0 (PREMIER Biosoft, San Francisco, CA, USA) and synthesized by BGI Tech Solutions (Beijing Liuhe, China). Primer sequences are listed in Table 1.

\begin{tabular}{ll}
\hline Table 1. Primers used for polymerase chain reaction amplification & Table 1. F \\
SNP & $\mathrm{F}$ \\
rs2104286 & $\mathrm{R}$ \\
rs12722489 & $\mathrm{F}$ \\
rs11594656 & $\mathrm{R}$ \\
rs1800896 & $\mathrm{F}$ \\
rs1800871 & $\mathrm{R}$ \\
rs1800872 & $\mathrm{F}$ \\
rs2430561 & $\mathrm{R}$ \\
& $\mathrm{F}$ \\
rs1799864 & $\mathrm{R}$ \\
rs2004640 & $\mathrm{F}$ \\
PCR: polymerase chain reaction; SNP: single nucleotide polymorphism; F: forward primer; R: reverse primer. & $\mathrm{R}$ \\
\hline
\end{tabular}

\section{Linkage disequilibrium analysis and haplotype construction}

The degree of linkage disequilibrium between the SNPs was analyzed using SHEsis software ${ }^{[7]}$ and expressed as $\mathrm{D}^{\prime}$ and $\mathrm{r}^{2}$, with larger values of $\mathrm{D}^{\prime}$ and $\mathrm{r}^{2}$ indicating a higher degree of linkage disequilibrium between the two associated SNPs. Values of $\mathrm{D}^{\prime}>0.9$ and $\mathrm{r}^{2}>0.33$ are generally considered to indicate highly interlocked genes. If D' [?] 0.8, the SNPs were considered to be sites for the construction of haplotypes; haplotype frequencies $<0.03$ were not included in the statistical analysis.

\section{Statistical analysis}

Statistical analysis was performed using SPSS 26.0 (International Business Machine Corp, Armonk, NY, USA). The frequencies of genotypes, alleles, and haplotypes between the two groups were analyzed by $\chi^{2}$ or Fisher's exact methods. Odds ratios (ORs) with $95 \%$ confidence intervals (CIs) were used to calculate the risk of susceptibility. The Hardy-Weinberg equilibrium was determined to assess the representativeness of the sample. $\mathrm{P}<0.05$ was considered statistically significant.

\section{Results}




\section{Genotype and allele frequencies}

Genotype distributions were in Hardy-Weinberg equilibrium, indicating that the subjects were representative of the general population. The frequencies of the genotypes and alleles of $I L 2 R A, I L-10, I \Phi N-\gamma, I P \Phi 5$, and CCR2 between EBV-HLH and control groups are shown in Table 2.

The IL2RA rs2104286 AA genotype and A allele were significantly more frequent in the EBV-HLH group than in the control group, suggesting that they might be associated with a significantly increased risk of EBV-HLH $(\mathrm{OR}=2.894,95 \% \mathrm{CI}=1.374-6.094$ and $\mathrm{OR}=2.077,95 \% \mathrm{CI}=1.232-3.502$, respectively $)$. However, the frequencies of theIL2RA rs12722489 and rs11594656 genotypes and alleles were similar in the EBV-HLH and control groups $(\mathrm{P}>0.05)$.

The $I L-10$ rs1800896 AA genotype and A allele were more frequent in the EBV-HLH group, suggesting that they might be associated with a significantly increased risk of EBV-HLH (OR $=3.792,95 \%$ CI $=1.779-$ 8.080 and $\mathrm{OR}=2.194,95 \% \mathrm{CI}=1.279-3.763$, respectively). The frequencies of $I L-10 \mathrm{rs} 1800872 \mathrm{AC}$ and CC genotypes and $\mathrm{C}$ allele were significantly higher in the EBV-HLH group, and the CC genotype and C allele were also associated with a significantly increased risk of EBV-HLH $(\mathrm{OR}=6.222,95 \% \mathrm{CI}=1.330-29.109$ and $\mathrm{OR}=2.176,95 \% \mathrm{CI}=1.257-3.767$,respectively). Meanwhile, the distribution of $I L-10 \mathrm{rs} 1800871$ was similar in both groups $(\mathrm{P}>0.05)$.

In addition, there were no significant differences in the frequencies of $I \Phi N-\gamma$ rs2430561 and IRF5 rs2004640 genotypes and alleles between the two groups $(\mathrm{P}>0.05)$ (Table 2 ).

Table 2. Genotype and allele frequencies in children with Epstein-Barr virus-associated hemophagocytic lyı Gene

ILRRA

$I L-10$ 
$I \Phi N-\gamma$

IRF5

CCR2

SNP: single nucleotide polymorphism values calculated from two-sided $\chi^{2}$ tests.

\section{Linkage disequilibrium analysis of identified SNPs}

The levels of linkage disequilibrium for IL2RA rs2104286, rs12722489, and rs11594656 and $I L-10$ rs1800896, rs1800871, and rs1800872 were analyzed using SHEsis software. There was a strong interlocking disequilibrium between IL2RA rs2104286 and rs12722489 ( $\left.\mathrm{D}^{\prime}=1, \mathrm{r}^{2}=0.311\right)$, and between $I L-10 \mathrm{rs} 1800871$ and rs1800872 ( $\left.D^{\prime}=1, \mathrm{r}^{2}=0.651\right)$ (Fig. 1). 


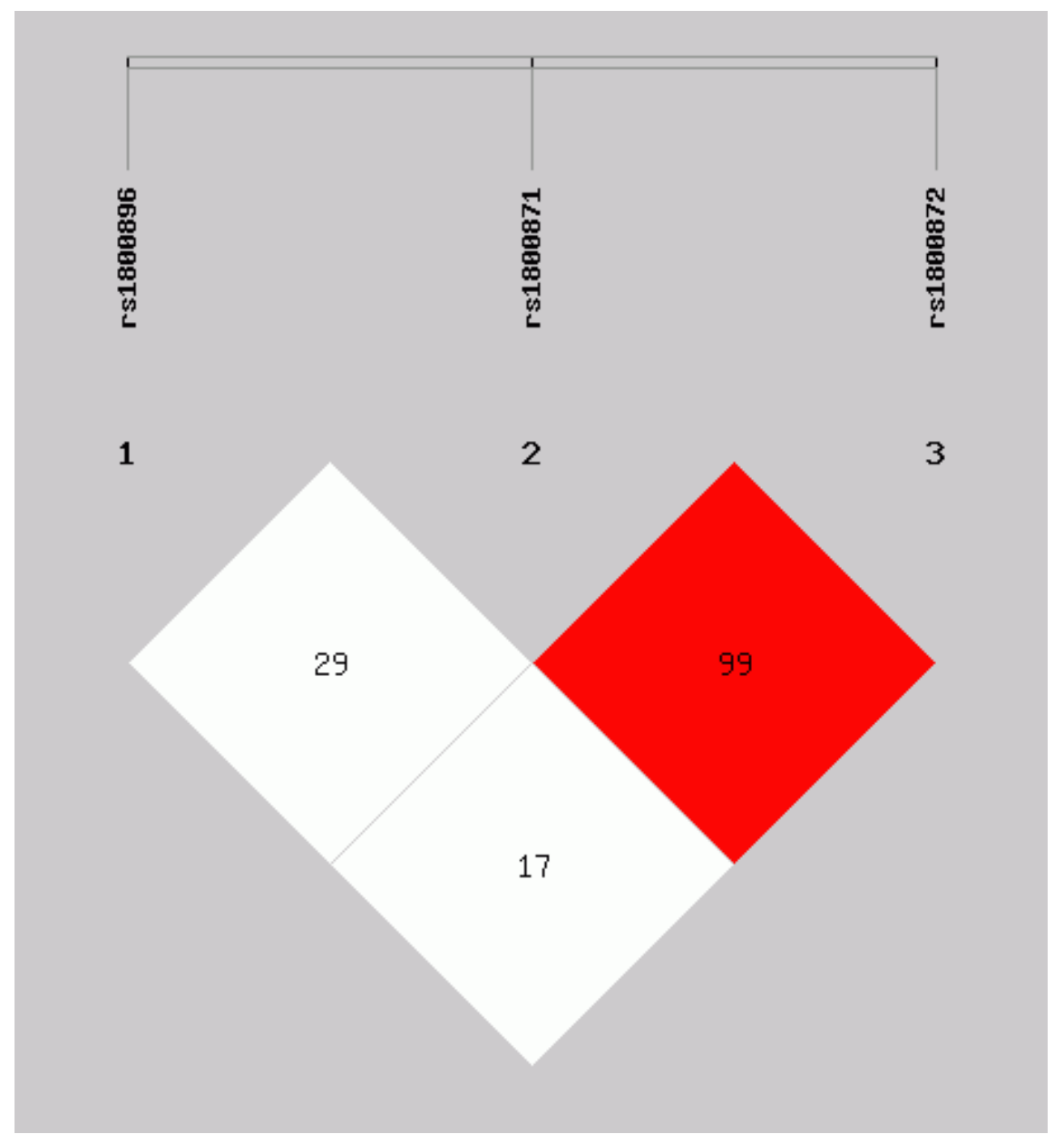

\section{Differences in the distributions of SNP haplotypes}

Haplotypes were constructed using SHEsis software in the order IL2RA rs2104286-rs12722489-rs11594656 and $I L$ 10rs1800896-rs1800871-rs1800872, respectively, and the distributions of the IL2RA and $I L-10$ haplotypes were compared between the EBV-HLH and control groups. As shown in Table 3, there were no significant differences in IL2RA AGA, GAA, GAT, and GGA between the two groups (P >0.05); however, the frequencies of AGT and GGT were significantly higher $(\mathrm{P}=0.006)$ and lower $(\mathrm{P}=0.001)$, respectively, in the EBV-HLH group. This suggested that IL2RA AGT was associated with an increased risk of EBV-HLH (OR $=2.102,95 \% \mathrm{CI}=1.229-3.593)$, while GGT significantly reduced the susceptibility to EBV-HLH $(\mathrm{OR}=$ $0.364,95 \% \mathrm{CI}=0.192-0.689$ ). There was no significant difference in the distribution of $I L-10$ ATA, GCA, or GCC haplotypes between the two groups $(\mathrm{P}>0.05)$. However, the frequency of $I L-10$ ACC was significantly higher in the EBV-HLH group $(\mathrm{P}=0.003$, $\mathrm{OR}=2.962,95 \% \mathrm{CI}=1.429-6.141)$, suggesting that this haplotype may be associated with an increased risk of HLH in children with EBV, while the significantly lower frequencies of ACA and GTA $(\mathrm{P}=0.020, \mathrm{OR}=0.253,95 \% \mathrm{CI}=0.073-0.876 ; \mathrm{P}<0.05, \mathrm{OR}=0.218,95 \%$ $\mathrm{CI}=0.093-0.513$, respectively) indicated that $I L-10 \mathrm{ACA}$ and GTA haplotypes may be protective factors for EBV-HLH.

Table 3. Haplotype frequencies in patients with Epstein-Barr virus-associated hemophagocytic lymphohisti Haplotype

IL2RA rs2104286-rs12722489-rs11594656

A G A 
A G T

G A A

G A T

G G A

G G T

$I L-10$ rs1800896-rs1800871-rs1800872

A C A

A C C

A T A

G C A

G C C

G T A

OR: odds ratio; CI: confidence interval; NA: not applicable. ${ }^{*} \mathrm{P} ; 0.05$.

\section{Discussion}

EBV-HLH is a typical cytokine storm syndrome, and cytokines play a crucial role in the pathogenesis of EBVHLH. Wada et al ${ }^{[8]}$. observed that EBV-infected patients had different serum cytokine levels and different severities of clinical manifestations. The synthesis, secretion, and biological effects of cytokines are regulated by their genes, and many studies have demonstrated important roles of cytokine gene polymorphisms in regulating cytokine levels and influencing the development of diseases ${ }^{[9-11]}$. SNPs are the most common form of gene polymorphisms, and we therefore hypothesized that cytokine SNPs might play an important role in the pathogenesis of and susceptibility to EBV-HLH.

Soluble IL-2 receptor (sIL-2R, also known as sCD25) is a marker of T-lymphocyte activation and is elevated in conditions such as infection, trauma, malignant hematological disorders, and autoimmune diseases. sIL$2 \mathrm{R}$ is currently used as an indicator of the severity of several diseases, and its level was shown to be directly proportional to the degree of inflammation ${ }^{[12]}$. Excessive activation of CD8+ T cells and significantly increased serum levels of sIL-2R have made sIL-2R an important diagnostic and disease marker for HLH in patients with EBV. The HLH-2004 diagnostic guidelines even added serum sIL-2R level [?]2400 U/ml as a new diagnostic criterion for HLH ${ }^{[13]}$. Furthermore, SNPs of the IL2RAgene encoding sIL-2R are associated with the development of autoimmune diseases, such as diabetes, multiple sclerosis, and baldness ${ }^{[4-16]}$.

In the current study, we investigated the three most common IL2RAloci, rs2104286, rs12722489, and rs11594656, and found that the rs2104286 AA genotype and A allele frequencies were significantly higher in children with EBV-HLH than in controls, suggesting that these might be risk factors for the development of EBV-HLH in children. rs2104286 is located in the first intron of the IL2RA gene, which is not a coding or regulatory region; however, this SNP has been associated with several autoimmune diseases, including multiple sclerosis and rheumatoid arthritis ${ }^{[17]}$. Carriers of the rs2104286 A allele have elevated sIL-2R levels, diminished IL-2R signaling, increased granulocyte-macrophage colony-stimulating factor production by memory CD4+ T cells, increased frequency of CD25+ naïve T cells, and decreased CD25 expression on the surface of memory CD127+CD25+ and Treg cells ${ }^{[15,18]}$. Moreover, the rs2104286 polymorphism can affect the activity of enhancer elements in the first intron of the IL2RA gene and the binding affinity of the transcription factor TRAF4, as well as mRNA processing and half-life, to influence IL2RA expression ${ }^{[17]}$. Dendrouet al. ${ }^{[19]}$ found that the rs2104286 AA genotype upregulated CD25 (IL-2R $\alpha$ ) expression levels on CD4+ naïve T cells and CD14+CD16+ monocytes, thereby enhancing T-cell activation, and this genotypic effect persisted after $\mathrm{T}$ cell activation, leading to a high proportion of CD69+CD4+ naïve $\mathrm{T}$ cells that upregulated CD25 in donors carrying the susceptible rs2104286 allele. We thus deduced that the rs2104286 polymorphism might affect the pathogenesis of EBV-HLH by upregulating CD25 expression on CD4+ naïve $\mathrm{T}$ cells, thus increasing sIL-2R levels.

IL-10 is produced by Th2 cells and is an important anti-inflammatory factor. Cellular secretion of IL-10 is significantly increased during microbial infections and autoimmune diseases. It is involved in the development 
of HLH by modulating immunity and suppressing the inflammatory response. Significantly elevated serum IL-10 levels are a characteristic cytokine pattern of $\mathrm{HLH}^{[20]}$. Sánchez et al. ${ }^{[21]}$ reported that certain SNPs in the promoter region of the $I L-10$ gene might influence IL-10 production and response intensity, and were associated with disease development. $I L-10$ SNPs were shown to be associated with various diseases, such as sepsis, asthma, multiple sclerosis, ankylosing spondylitis, gastric cancer, and coronary heart disease ${ }^{[4,21,22]}$. In the current study, we selected rs1800896, rs1800871, and rs1800872 for testing and showed that the frequencies of the AA genotype and A allele of rs1800896 and the CC genotype and C allele of rs1800872 were significant higher in children with EBV-HLH than in controls, suggesting that these might be predisposing risk factors for the development of EBV-HLH in children. Wang et al . ${ }^{[9]}$ studied the relationship between the rs1800872 and children with EBV-HLH, and similarly showed that the rs1800872 CC genotype and C allele frequencies were higher in children with EBV-HLH than in patients with infectious mononucleosis (IM) and in healthy controls. rs1800896 is located at a putative ETS-like transcription factor-binding site and rs1800872 at a putative signal transducer and activator of transcription 3-binding site and regions of negative regulatory function ${ }^{[23,24]}$. Polymorphisms at these sites may thus influence transcription factor binding and consequent $I L-10$ transcription and IL-10 serum levels, thus affecting disease susceptibility ${ }^{[25,26]}$. IL-10 levels are significantly elevated in children with EBV-HLH. Numerous studies have shown that the $\mathrm{C}$ allele of rs1800872 is associated with high levels of ${ }^{[27,28]}$, while the relationships between the $\mathrm{G}$ and $\mathrm{A}$

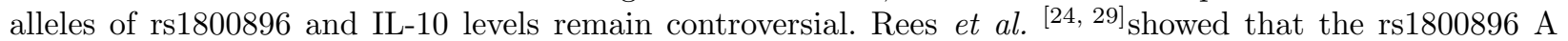
allele enhanced transcription of the $I L-10$ gene and resulted in high levels of IL-10. In contrast, Engelhardt et al. ${ }^{[30-32]}$ showed that the rs1800896 G allele was associated with higher IL-10 production, while the rs1800896 A allele resulted in lower IL-10 production. The frequencies of $I L-10$ polymorphisms differ among populations ${ }^{[33]}$, and IL-10 plays different roles in different diseases, although its regulatory mechanisms are not fully understood. Numerous genetic correlation studies and a few functional studies have shown that the regulation of IL-10 is complex and multifactorial. Further studies are therefore needed to determine how rs1800896 and rs1800872 regulate the production of IL-10 and affect the susceptibility to and clinical course of EBV-HLH in children. In addition, the rs1800896 polymorphism can also influence EBV infection status. Consistent with the results of the present study, Helminenet al. ${ }^{[34]}$ also showed that individuals carrying the rs1800896 A allele were more susceptible to severe EBV infection. The rs1800896 and rs1800872 polymorphisms may thus influence EBV-HLH susceptibility by synergistically regulating IL-10 expression and EBV infection status.

Haplotype analysis provides more accurate genetic information than analysis of individual SNPs. Comparisons between children with EBV-HLH and controls based on estimated haplotype frequencies thus provide a better understanding of the role of cytokine SNPs in EBV-HLH susceptibility. The present results showed that the frequency of theIL2RA AGT (rs2104286-rs12722489-rs11594656) haplotype was significantly increased while that of the GGT haplotype was significantly decreased in children with EBV-HLH. The frequency of the $I L-10$ ACC (rs1800896-rs1800871-rs1800872) haplotype was also significantly increased, whereas the frequencies of the ACA and GTA haplotypes were significantly decreased. In addition, some of these haplotypes showed strong linkage disequilibrium, suggesting that $I L 2 R A$ and $I L-10$ haplotype association and linkage disequilibrium may play important roles in the susceptibility to EBV-HLH. The results of this study suggest that the IL2RA AGT haplotype may increase the risk of EBV-HLH; however, no other studies have simultaneously tested the IL2RA rs2104286, rs12722489, and rs11594656 loci, and studies of the AGT haplotype in other diseases are lacking, and further studies are therefore needed to validate the roles of the AGT and GGT haplotypes. The common $I L$-10rs1800896-rs1800871-rs1800872 haplotypes are GCC, ACC, and ATA. The ACC haplotype is associated with moderate IL-10 production ${ }^{[35]}$, but its role in different diseases is controversial. Marangon et al. ${ }^{[36]}$ showed that this haplotype was a protective factor in diffuse large B-cell lymphoma, while Gao et al. ${ }^{[28]}$ showed that it was associated with an increased risk of IgA nephropathy.

We also analyzed $I \Phi N-\gamma$ rs2430561, IRF5 rs2004640, and CCR2 rs2004640, but found no significant associations between these SNPs and susceptibility to EBV-HLH. IFN- $\gamma$ is an important cytokine produced by a variety of immune cells in response to inflammatory stimuli. It regulates the body's immune response, and is significantly elevated in children with EBV-HLH. $I \Phi N-\gamma$ rs2430561 has been shown to affect susceptibility 
to diseases such as systemic lupus erythematosus, sepsis, ankylosing spondylitis, and breast cancer ${ }^{[37-40]}$, but no previous association between this SNP and HLH has been reported. IRF5 is a member of the IRF transcription factor family that plays important roles in both the interferon pathway and the Toll-like receptor signaling pathway, and is closely associated with many autoimmune diseases ${ }^{[41,42]}$. IRF5 rs2004640 has been associated with susceptibility to diseases such as systemic juvenile idiopathic arthritis(sJIA), systemic lupus erythematosus(SLE), and systemic sclerosis(SS ${ }^{[43,44]}$. Yanagimachi et al. ${ }^{[42]}$ investigated the relationship between rs2004640 and susceptibility to secondary HLH, such as macrophage activation syndrome and EBV-HLH, and found that the GT/TT genotype at this locus increased overall susceptibility to secondary HLH, but not to EBV-HLH, consistent with the results of the present study. The chemokine receptor CCR2 plays a key role in several diseases, and the CCR2rs1799864 gene polymorphism has also been associated with susceptibility to diseases such as ischemic stroke and multiple sclerosis ${ }^{[45,46]}$. Ou et al ${ }^{[47]}$ found no significant difference in the rs1799864 polymorphism between children with and without HLH, suggesting that this locus may be independent of HLH susceptibility, consistent with the current study. However, the paucity of relevant studies and the limited numbers of cases mean that further studies are needed to confirm the relationships between the above genetic polymorphisms and EBV-HLH.

This study had some limitations. First, the relatively small sample size may have limited the statistical power, and more multicenter studies with larger sample sizes are therefore needed to validate our findings. Second, because of technical limitations in the quantitative analysis of cytokines before 2014, cytokine profiles were not available for more than half of the patients. Unfortunately, we were therefore unable to analyze the relationships among SNPs, cytokine secretion, and HLH.

\section{Conclusions}

This study analyzed the associations between polymorphisms and haplotypes of $\sigma I \Lambda-2 P, I \Lambda-10, I \Phi N-\gamma, I P \Phi 5$ , and CCR2 and susceptibility to EBV-HLH in children. The IL2RA rs2104286 AA genotype and A allele, $I L-10$ rs1800896 AA genotype and A allele, and $I L-10$ rs1800872 CC genotype and C allele, as well as theIL2RA AGT and $I L-10$ ACC haplotypes were identified as possible susceptibility risk factors for HLH in children with EBV.

\section{Data Availability Statement}

The datasets for this article are not publicly available due to concerns regarding participant/patient anonymity. Requests to access the datasets should be directed to the corresponding author.

\section{Ethics Statement}

The studies involving human participants were reviewed and approved by the Ethics Committee of the Seventh Affiliated Hospital of Sun Yat-Sen University, Sun Yat-Sen Memorial Hospital of Sun Yat-Sen University and Huizhou First Hospital. Written informed consent to participate in this study was provided by the participants' legal guardian/next of kin.

\section{Author Contributions}

HX and GC designed the experiments and drafted the manuscript. NT and JH accomplished works of the data collection, DNA extraction, and genotyping. CC and XW helped to enroll all of the patients. HX guided to conduct the statistic works. All authors contributed to the article and approved the submitted version.

\section{Funding}

This study was supported by Sanming Project of Medicine in Shenzhen (SZSM202011004), Science,Technology and Innovation Commission of Shenzhen Municipality( JCYJ20190809160609727).

\section{Conflict of Interest}


The authors declare that the research was conducted in the absence of any commercial or financial relationships that could be construed as a potential conflict of interest.

\section{Acknowledgments}

The authors thank all the patients and their families. The authors also thank the support and advise from Doctor Junchao Dong, Department Immunology, Zhongshan School of Medicine, Sun Yat-sen University, Guangzhou, Guangdong, China.

\section{References}

[1]M. Madkaikar, et al. Current Updates on Classification, Diagnosis and Treatment of Hemophagocytic Lymphohistiocytosis (HLH)[J]. Indian J Pediatr, 2016, 83(5): 434-443

[2]S. Bhushan,N. B. Perumal. Disease associated cytokine SNPs database: an annotation and dissemination model[J]. Cytokine, 2012, 57(1): 107-112

[3]A. P. Kallaur, et al. Genetic, Immune-Inflammatory, and Oxidative Stress Biomarkers as Predictors for Disability and Disease Progression in Multiple Sclerosis[J]. Mol Neurobiol, 2017, 54(1): 31-44

[4]M. A. Al-Naseri, et al. Association between interleukin-4 and interleukin-10 single nucleotide polymorphisms and multiple sclerosis among Iraqi patients[J]. Neurol Sci, 2019, 40(11): 2383-2389

[5]Y. H. Chang, et al. Tumor necrosis factor alpha promoter polymorphism associated with increased susceptibility to secondary hemophagocytic lymphohistiocytosis in the Korean population[J]. Cytokine, 2006, 36(1-2): $45-50$

[6]E. Bergsten, et al. Confirmed efficacy of etoposide and dexamethasone in HLH treatment: long-term results of the cooperative HLH-2004 study[J]. Blood, 2017, 130(25): 2728-2738

[7]Y. Y. Shi,L. He. SHEsis, a powerful software platform for analyses of linkage disequilibrium, haplotype construction, and genetic association at polymorphism loci[J]. Cell Res, 2005, 15(2): 97-98

[8]T. Wada, et al. Cytokine profiles in children with primary Epstein-Barr virus infection[J]. Pediatr Blood Cancer, 2013, 60(7): E46-48

[9]Y. Wang, et al. IL-10-592 A/C polymorphisms is associated with EBV-HLH in Chinese children[J]. Hematology, 2016, 21(2): 95-98

[10]P. Yang, et al. Associations between Seven Common Cytokine Gene Polymorphisms and Coronary Artery Disease: Evidence from a Meta-Analysis[J]. Int Arch Allergy Immunol, 2020, 181(4): 301-310

[11]C. A. D. de Lima, et al. Are key cytokines genetic and serum levels variations related to rheumatoid arthritis clinical severity?[J]. Gene, 2020, 722: 144098

[12]M. Lin, et al. Clinical utility of soluble interleukin-2 receptor in hemophagocytic syndromes: a systematic scoping review[J]. Ann Hematol, 2017, 96(8): 1241-1251

[13]J. I. Henter, et al. HLH-2004: Diagnostic and therapeutic guidelines for hemophagocytic lymphohistiocytosis[J]. Pediatr Blood Cancer, 2007, 48(2): 124-131

[14]Y. Okada, et al. Genetics of rheumatoid arthritis contributes to biology and drug discovery[J]. Nature, 2014, 506(7488): 376-381

[15]S. Buhelt, et al. Relationship between Multiple Sclerosis-Associated IL2RA Risk Allele Variants and Circulating T Cell Phenotypes in Healthy Genotype-Selected Controls[J]. Cells, 2019, 8(6)

[16]A. Alcina, et al. IL2RA/CD25 gene polymorphisms: uneven association with multiple sclerosis (MS) and type 1 diabetes (T1D)[J]. PLoS One, 2009, 4(1): e4137 
[17]X. X. Wang,T. Chen. Meta-analysis of the association of IL2RA polymorphisms rs2104286 and rs12722489 with multiple sclerosis risk[J]. Immunol Invest, 2018, 47(5): 431-442

[18]K. Cerosaletti, et al. Multiple autoimmune-associated variants confer decreased IL-2R signaling in CD4+ CD25(hi) T cells of type 1 diabetic and multiple sclerosis patients[J]. PLoS One, 2013, 8(12): e83811

[19]C. A. Dendrou, et al. Cell-specific protein phenotypes for the autoimmune locus IL2RA using a genotypeselectable human bioresource[J]. Nat Genet, 2009, 41(9): 1011-1015

[20]Y. Tang, et al. Early diagnostic and prognostic significance of a specific Th1/Th2 cytokine pattern in children with haemophagocytic syndrome[J]. Br J Haematol, 2008, 143(1): 84-91

[21]R. Posadas-Sánchez, et al. The IL-10-1082 (rs1800896) G allele is associated with a decreased risk of developing premature coronary artery disease and some IL-10 polymorphisms were associated with clinical and metabolic parameters. The GEA study[J]. Cytokine, 2018, 106: 12-18

[22]M. N. Karimabad, et al. Is the IL-10 promoter polymorphism at position -592 associated with immune system-related diseases?[J]. Inflammation, 2013, 36(1): 35-41

[23]J. H. Jung, et al. Association of Interleukin 10 Gene Polymorphisms with Autoimmune Thyroid Disease: Meta-Analysis[J]. Scand J Immunol, 2016, 84(5): 272-277

[24]L. E. Rees, et al. The interleukin-10-1082 G/A polymorphism: allele frequency in different populations and functional significance[J]. Cell Mol Life Sci, 2002, 59(3): 560-569

[25]J. Zheng, et al. IL-10, IL-18 Gene Polymorphisms Might Influence Predisposition to Coronary Artery Disease in East Asians: A Meta-Analysis[J]. Immunol Invest, 2020: 1-10

[26]A. J. Smith,S. E. Humphries. Cytokine and cytokine receptor gene polymorphisms and their functionality[J]. Cytokine Growth Factor Rev, 2009, 20(1): 43-59

[27]M. Pastuszczak, et al. Association of interleukin-10 promoter polymorphisms with serofast state after syphilis treatment[J]. Sex Transm Infect, 2019, 95(3): 163-168

[28]J. Gao, et al. Association of Interleukin-10 Polymorphisms (rs1800872, rs1800871, and rs1800896) with Predisposition to IgA Nephropathy in a Chinese Han Population: A Case-Control Study[J]. Kidney Blood Press Res, 2017, 42(1): 89-98

[29]R. Nasiri, et al. Gene polymorphisms of interleukin-10 and transforming growth factor beta in allergic rhinitis[J]. Allergol Immunopathol (Madr), 2016, 44(2): 125-130

[30]K. R. Engelhardt,B. Grimbacher. IL-10 in humans: lessons from the gut, IL-10/IL-10 receptor deficiencies, and IL-10 polymorphisms[J]. Curr Top Microbiol Immunol, 2014, 380: 1-18

[31]M. Korppi, et al. IL-10 gene polymorphism is associated with preschool atopy and early-life recurrent wheezing after bronchiolitis in infancy[J]. Pediatr Pulmonol, 2017, 52(1): 14-20

[32]S. Mohammadi, et al. Interleukin 10 gene promoter polymorphisms (rs1800896, rs1800871 and rs1800872) and haplotypes are associated with the activity of systemic lupus erythematosus and IL10 levels in an Iranian population[J]. Int J Immunogenet, 2019, 46(1): 20-30

[33]P. Hong, et al. Associations between genetic polymorphisms in interleukin-10 and hematological oncology: evidence from a meta-analysis[J]. Cancer Biol Ther, 2020, 21(4): 372-378

[34]M. Helminen, et al. Polymorphism of the interleukin-10 gene is associated with susceptibility to EpsteinBarr virus infection[J]. J Infect Dis, 1999, 180(2): 496-499

[35]P. Kapelski, et al. Family-based association study of interleukin 10 (IL10) and interleukin 10 receptor alpha (IL10RA) functional polymorphisms in schizophrenia in Polish population[J]. J Neuroimmunol, 2016, 297: 92-97 
[36]A. V. Marangon, et al. Impact of SNPs/Haplotypes of IL10 and IFNG on the Development of Diffuse Large B-Cell Lymphoma[J]. J Immunol Res, 2019, 2019: 2137538

[37]H. D. da Silva, et al. Interferon gamma and Interleukin 10 polymorphisms in Brazilian patients with systemic lupus erythematosus[J]. Mol Biol Rep, 2014, 41(4): 2493-2500

[38]C. J. Li, et al. Correlations of IFN- $\gamma$ genetic polymorphisms with susceptibility to breast cancer: a meta-analysis[J]. Tumour Biol, 2014, 35(7): 6867-6877

[39]D. Wang, et al. Functional polymorphisms of interferon-gamma affect pneumonia-induced sepsis[J]. PLoS One, 2014, 9(1): e87049

[40]H. Xu,B. Li. Effect of Interferon- $\gamma$ Polymorphisms on Ankylosing Spondylitis: A Case-Control Study[J]. Med Sci Monit, 2017, 23: 4126-4131

[41]S. E. Löfgren, et al. Promoter insertion/deletion in the IRF5 gene is highly associated with susceptibility to systemic lupus erythematosus in distinct populations, but exerts a modest effect on gene expression in peripheral blood mononuclear cells[J]. J Rheumatol, 2010, 37(3): 574-578

[42]M. Yanagimachi, et al. Association of IRF5 polymorphisms with susceptibility to hemophagocytic lymphohistiocytosis in children[J]. J Clin Immunol, 2011, 31(6): 946-951

[43]G. B. Nordang, et al. Interferon regulatory factor 5 gene polymorphism confers risk to several rheumatic diseases and correlates with expression of alternative thymic transcripts[J]. Rheumatology (Oxford), 2012, 51(4): $619-626$

[44]Y. Xu, et al. Polymorphisms in STAT4 and IRF5 increase the risk of systemic sclerosis: a meta-analysis[J]. Int J Dermatol, 2016, 55(4): 408-416

[45]N. Zhao, et al. Association of inflammatory gene polymorphisms with ischemic stroke in a Chinese Han population[J]. J Neuroinflammation, 2012, 9: 162

[46]J. Javor, et al. The $+190 \mathrm{G} / \mathrm{A}$ (rs1799864) polymorphism in the C-C chemokine receptor 2 (CCR2) gene is associated with susceptibility to multiple sclerosis in HLA-DRB1*15:01-negative individuals[J]. J Neurol Sci, 2015, 349(1-2): 138-142

[47]D. Y. Ou, et al. Association of CCR2 gene rs1799864 polymorphism with hemophagocytic lymphohistiocytosis in children[J]. Zhongguo Dang Dai Er Ke Za Zhi, 2015, 17(2): 164-167

\section{Hosted file}

Table 1.pdf available at https://authorea.com/users/362314/articles/483521-polymorphismsand-haplotypes-of-il2ra-il-10-ifn-\%CE\%B3-irf5-and-ccr2-are-associated-with-epsteinbarr-virus-associated-hemophagocytic-lymphohistiocytosis-in-children

\section{Hosted file}

Table 2.pdf available at https://authorea.com/users/362314/articles/483521-polymorphismsand-haplotypes-of-il2ra-il-10-ifn-\%CE\%B3-irf5-and-ccr2-are-associated-with-epsteinbarr-virus-associated-hemophagocytic-lymphohistiocytosis-in-children 


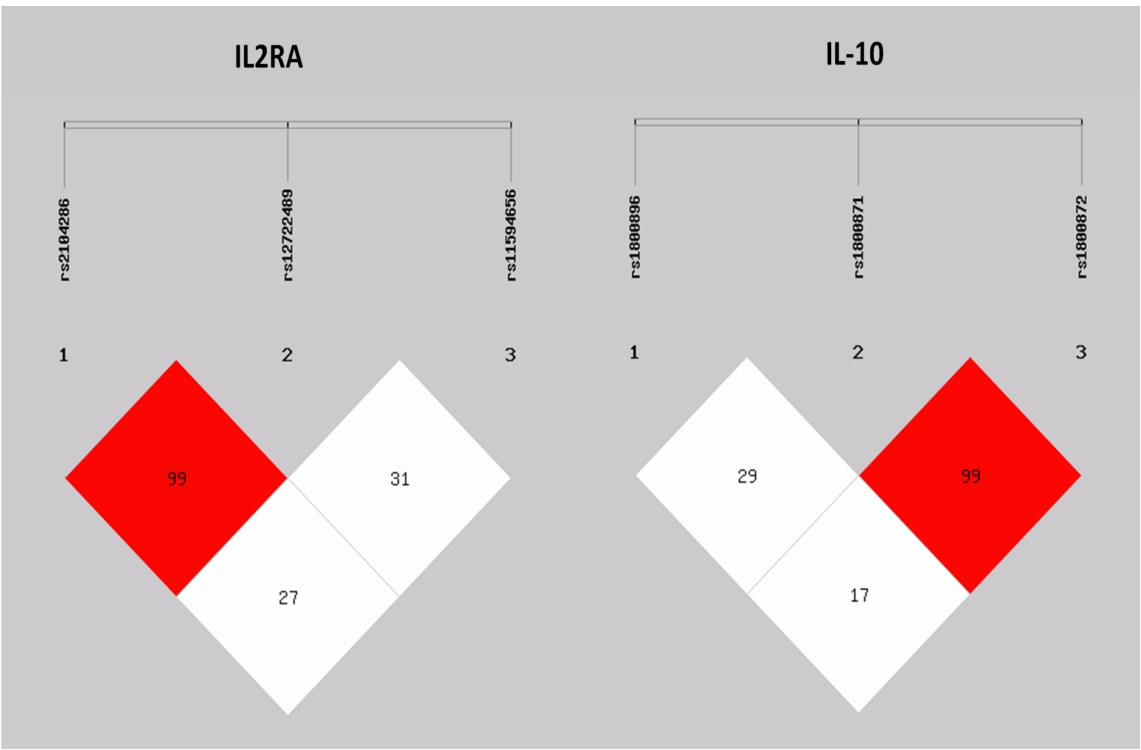

\section{Hosted file}

Table 3.pdf available at https://authorea.com/users/362314/articles/483521-polymorphismsand-haplotypes-of-il2ra-il-10-ifn-\%CE\%B3-irf5-and-ccr2-are-associated-with-epsteinbarr-virus-associated-hemophagocytic-lymphohistiocytosis-in-children 